\title{
Solitary Fibrous Tumor of the Male Breast: A Case Report with Imaging Findings
}

\author{
Ho Suk Song, ${ }^{1}$ Ji Young Lee, ${ }^{1,}$ Su Young Kim, ${ }^{1}$ Byung Hoon Lee, ${ }^{1}$ You Sung Kim, ${ }^{1}$ Jin Woo Kim, ${ }^{1}$ Han \\ Seong Kim, ${ }^{2}$ and Jae Il Kim ${ }^{3}$ \\ ${ }^{1}$ Department of Radiology, Ilsan Paik Hospital, Inje University College of Medicine, Goyang, Republic of Korea \\ ${ }^{2}$ Department of Pathology, Ilsan Paik Hospital, Inje University College of Medicine, Goyang, Republic of Korea \\ ${ }^{3}$ Department of Surgery, Ilsan Paik Hospital, Inje University College of Medicine, Goyang, Republic of Korea \\ Corresponding author: Ji Young Lee, Department of Radiology, Ilsan Paik Hospital, Inje University College of Medicine, 2240, Daehwa-dong, Ilsanseo-gu, Goyang-si, \\ Gyeonggi-do 411-706, Republic of Korea. Tel: +82-319107689, Fax: +82-319107369, E-mail: drleeji@paik.ac.kr
}

Received 2017 December 27; Revised 2018 April 13; Accepted 2018 April 17.

\begin{abstract}
Solitary fibrous tumor (SFT) is an uncommon neoplasm of mesenchymal origin, and it most often occurs in the pleura. Mammary gland involvement is extremely rare in this disease. Herein, we report a case of SFT that presented as a slowly growing large mass in the breast of a 64-year-old man. On ultrasonographic examination, the mass was oval and circumscribed with heterogeneous echogenicity and increased intralesional blood flow. Moreover, the mass showed hard elasticity as revealed by shear-wave elastography (SWE), which has not been reported in English literature so far. We also briefly review the literature on the radiologic findings. Because of the nonspecific imaging findings and difficulty in its differentiation from other soft tissue tumors, complete excision with detailed immunohistochemistry is essential.
\end{abstract}

Keywords: Solitary Fibrous Tumor, Breast, Ultrasonography, Immunohistochemistry

\section{Introduction}

Solitary fibrous tumor (SFT), an uncommon neoplasm that has been found most commonly in the pleura but may also arise at various extrapleural sites, including the head, neck, lung, abdomen, pelvis, and extremities was first described as a primary spindle-cell tumor of the pleura by Klemperer and Rabin in $1931(1,2)$. SFT in the breast is very rare; only 23 cases have been reported in the medical literature (3-9). Herein, we report a case of breast SFT in a 64-yearold man. We describe the ultrasonographic features of this rare entity in addition to the elastographic findings, which have not been reported in the English literature so far.

\section{Case Presentation}

A 64-year-old man presented to our hospital with a painless breast mass. It had been palpable for 10 years, but gradually increased in size over the last two years, causing breast discomfort. He was taking diabetes medication and had no other remarkable medical history or family history of breast cancer. On physical examination, a firm nontender mass measuring about $5.0 \times 2.0 \mathrm{~cm}$ was noted in the upper inner quadrant of the left breast without overlying skin color change or palpable axillary lymph node. Breast ultrasonography (US) revealed a large solid mass in the upper inner breast $1 \mathrm{~cm}$ from the nipple. The mass was oval in shape, with circumscribed margins, and had heterogeneous echo pattern with internal vascularity on color Doppler sonography (Figure $1 \mathrm{~A}$ and $\mathrm{B}$ ). The mass showed hard elasticity as revealed by shear-wave elastography (SWE) (Figure 1C). Small gynecomastia was observed in the left retroareolar area. The patient had undergone lung cancer screening with low dose chest computed tomography (CT) scan without contrast enhancement a year previously, and the mass had been observed as an isodense solid mass in the left breast (Figure 1D). The long diameter of the mass was slightly increased compared to that observed during the previous screening CT. Considering the elastographic features and size interval change, the lesion was classified as breast imaging and reporting data systems (BI-RADS) category 4. We performed ultrasound-guided core biopsy using a 14-gauge needle. On histological examination, the biopsied tissue revealed fascicles of spindle cells separated by hyalinized collagen bands, indicat- 
ing the possibility of myofibroblastoma (MFB). The patient underwent complete surgical excision of the mass. On microscopic examination, the resected tumor was composed of bland-looking spindle cells that were arranged haphazardly in the fibrohyaline stroma resembling a keloid (Figure 2A). Immunohistochemical staining showed immunoreactivity for $\mathrm{CD} 34$ (Figure $2 \mathrm{~B}$ ), and the cells were negative for S100-protein. On the basis of microscopic findings and immunohistochemical analysis, the final diagnosis was SFT of the breast. The patient had an unremarkable postoperative course.

\section{Discussion}

SFT is a rare mesenchymal neoplasm accounting for $<$ $2 \%$ of all soft tissue tumors (3). It most commonly presents during the fourth to seventh decades of life with equal incidence rates by sex (4). It has been known to occur most frequently in the pleural cavity, but it has also been described in other sites - such as the retroperitoneum, abdominal cavity, proximal extremity, and head and neck which could have occurred owing to the increased recognition of this entity (3). Among the extrapleural sites, SFTs in the breast have only been reported in 23 cases, which is rare (3-9). Moreover, involvement of the male breast is rarer, and only a few cases have been reported $(4,5)$.

The typical clinical feature of breast SFT is a slowly enlarging nontender mass, and the symptoms differ depending on the location. When the SFT is in the lungs, chest pain and cough may arise. Abdominal pain and distension may be present when the SFT grows in the abdominal cavity. Intracranial SFT can cause headaches, while no other special symptom except a palpable mass is present in a chest wall SFT (10). This was also observed in our case.

The known ultrasonographic features of breast SFT are nonspecific and similar to a benign solid mass, such as a well-circumscribed, oval-shaped, isoechoic, or hypoechoic mass with vascularity (3-8). In our case, the breast SFT was depicted as well-circumscribed, oval-shaped, and heterogeneously hypoechoic mass with peripheral vascularity on color Doppler US.

Elastography, a method for imaging tissue stiffness, is the most noteworthy of the new technologies in recent diagnostic ultrasound systems that improve the specificity for solid masses in the breast. It can help differentiate between benign and malignant masses. According to Barr RG et al., who published the ultrasound elastography guideline, if BI-RADS 3 lesion has characteristics of a malignancy on elastography (strain or shear-wave), such as high stiff- ness, the lesion should be upgraded and biopsied (11). In our case, the mass showed high shear wave speed colorcoding red on the SWE. It was upgraded to BI-RADS 4; therefore, it had to be biopsied. To the best of our knowledge, there have been no articles describing elastographic findings of the breast SFT.

SFT can be hypodense or hyperdense, with respect to muscle depending on the collagen content, with heterogeneous enhancement on CT. On magnetic resonance image (MRI), the SFT is usually isointense on T1-weighted images and variable on T2-weighted images with vigorous enhancement (2). Because of these nonspecific radiologic findings and rarity of the tumor, it is difficult to distinguish SFT from other benign soft tissue tumors. Therefore, the exact diagnosis is usually made after surgical resection and immunohistochemical analysis.

Histologically, SFTs have fibroblast-like spindle-shaped cells and collagen bands. They have high vascularity and a tendency to undergo myxoid degeneration. Differential diagnosis of SFTs includes other bland-looking monomorphic spindle cell lesions in the breast, such as MFB, fibromatosis, nodular fasciitis, hemangiopericytomas, and benign peripheral nerve sheath tumors (8). Among these, MFBs and SFTs share many histological findings, which makes it difficult to differentiate them from each other. However, MFBs have immunoreactivity to muscle antigens, such as a-SMA and desmin, while SFTs have immunoreactivity to CD34 (9). In our case, the pathologic result by core needle biopsy was MFB, but the final diagnosis was SFT based on the detailed immunohistochemical examination. Therefore, small tissue sampling by core biopsy alone may have limited diagnostic value for this rare entity. Recently, nuclear expression of STAT6 (signal transducer and activator of transcription 6, interleukin-4 induced) has been found to a highly sensitive and almost perfectly specific immunohistochemical marker for SFT, and it can be helpful to distinguish this tumor type from histological mimics (12).

Although most SFTs are benign lesions, approximately $10 \%-20 \%$ of SFTs are malignant, and even benign SFTs have indeterminate malignant potential. Malignant SFTs are correlated with local recurrence and metastasis and the risk of metastasis has been reported to be as high as 25\% (3, 4). Histologically malignant lesions are characterized by hypercellularity, with moderate to marked atypia, necrosis, and mitotic activity of more than four mitoses per 10 high-power fields and infiltrative margins (3). Up to now, two malignant breast SFTs have been reported in the literature. These showed clear and focal lobulated boundary 

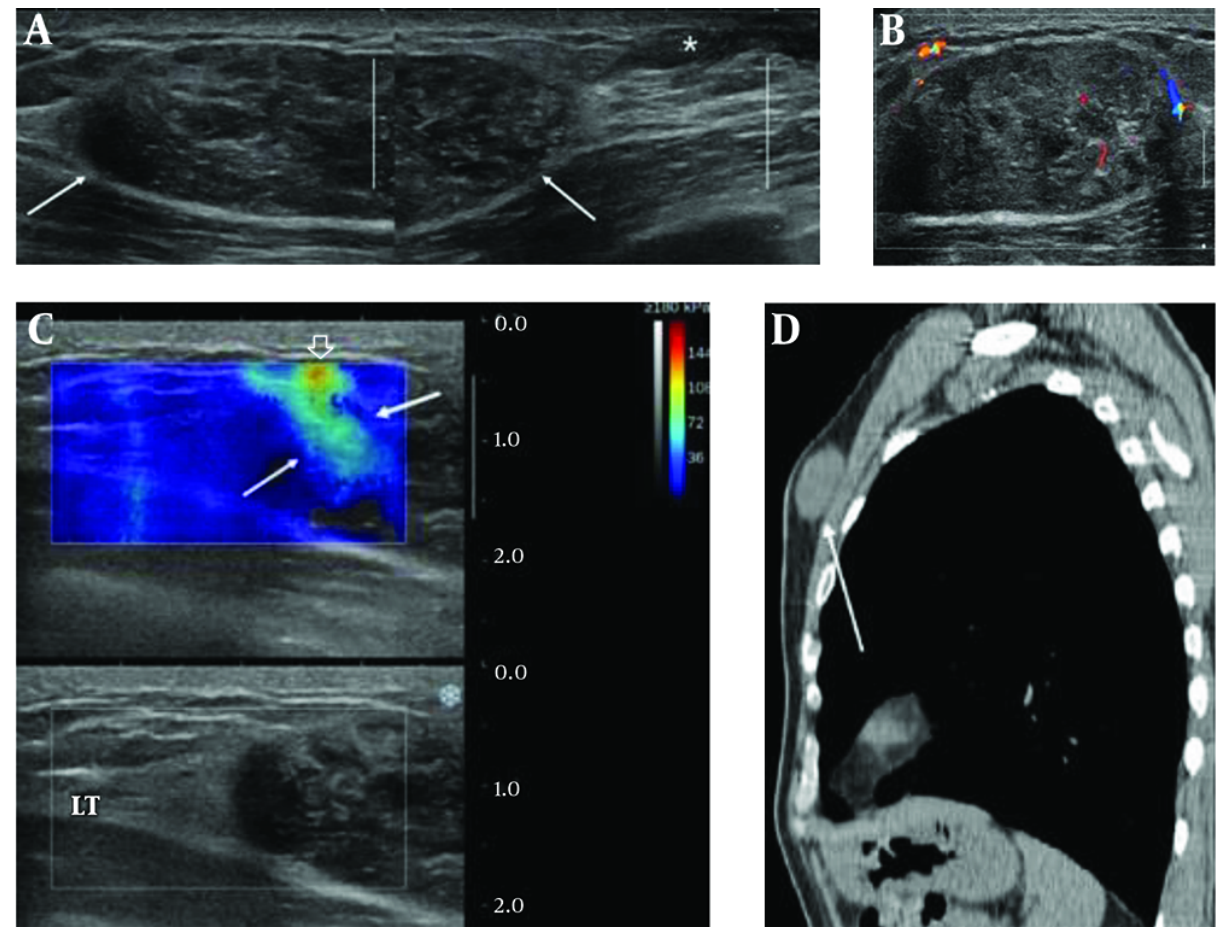

Figure 1. A 64-year-old man who presented with a painless palpable mass in the left breast. A, Transverse conventional B-mode US image shows a solid mass measuring $4.0 \times$ $1.6 \mathrm{~cm}$ with oval shaped, heterogeneously hypoechoic echotexture and well-defined margin (arrows) and small gynecomastia is noted (asterisk). B, The mass has peripheral and intralesional vascularities on color Doppler sonography. C, The mass shows color-coding red in the stiffest part of the mass (open arrow), mixed with light blue (arrows) on shear-wave elastography. D, The sagittal reconstruction of low dose non-enhanced chest CT scan for health checkup that was performed a year ago shows a well-defined oval-shaped solid mass, isodense to the muscle (arrow).
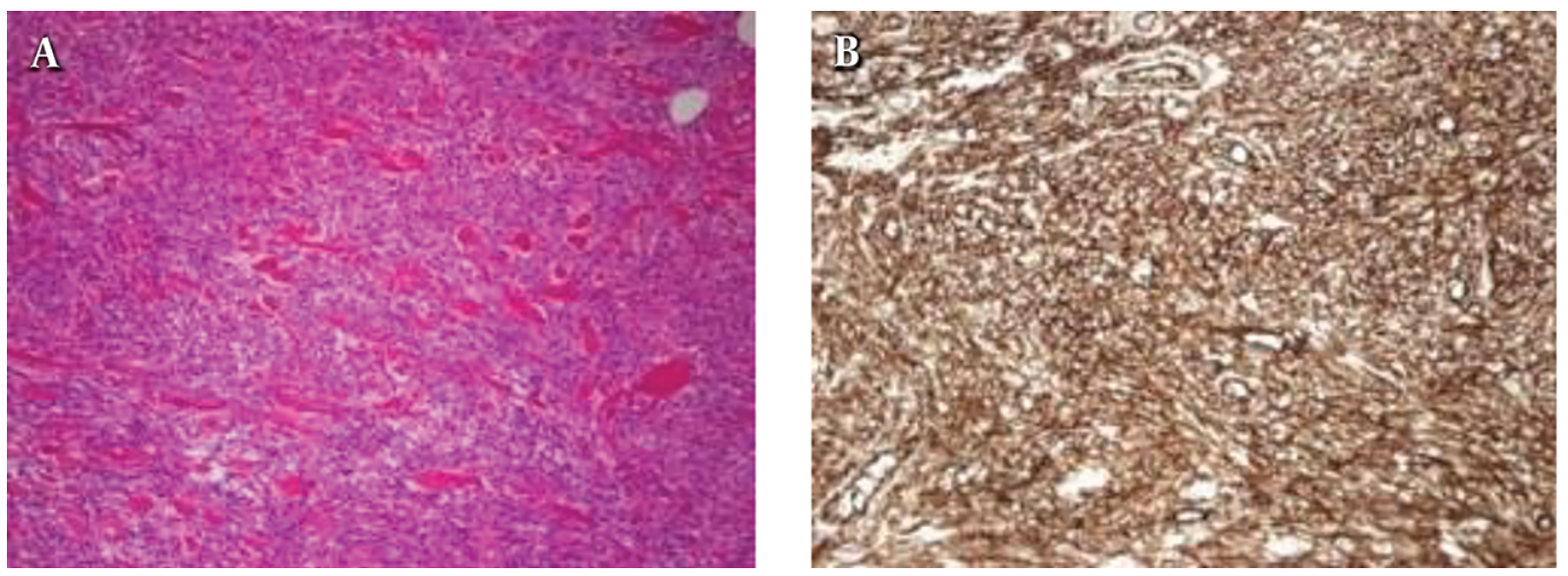

Figure 2. Photomicrographs of the excised mass. A, Bland-looking spindle cells are arranged haphazardly in the fibrohyaline stroma resembling a keloid (H\&E stain, $\times 100)$. $B$, Spindle cells are strongly positive for CD34 immunohistochemical stains $(\times 200)$.

on mammography or well-defined margin with pathologic uptake on positron emission tomography with 2-deoxy2-(fluorine-18) fluoro-D-glucose integrated with computed tomography $(7,13)$.
In conclusion, we presented a case of breast SFT with a well-defined heterogeneously hypoechoic mass with intralesional blood flows on color Doppler sonography that showed hard elasticity on SWE. Because of the difficulty in 
its differentiation from other soft tissue tumors due the nonspecific imaging findings and its unpredictable behavior, complete surgical resection with clear margins should be performed, and immunohistochemistry is essential for exact diagnosis.

\section{Footnotes}

Authors' Contributions: None declared.

Financial Disclosure: No funding support or grant was received for this study.

Funding/Support: None declared.

\section{References}

1. Klemperer P, Coleman BR. Primary neoplasms of the pleura. A report of five cases. Am J Ind Med. 1992;22(1):1-31. doi: 10.1002/ajim.4700220103. [PubMed: 1415270].

2. Ginat DT, Bokhari A, Bhatt S, Dogra V. Imaging features of solitary fibrous tumors. AJR Am J Roentgenol. 2011;196(3):487-95. doi: 10.2214/AJR.10.4948. [PubMed: 21343490].

3. Salemis NS. Solitary fibrous tumor of the breast: A case report and the review of the literature. Breast J. 2018;24(1):78-81. doi: 10.111//tbj.12841. [PubMed: 28621469].

4. Rhee SJ, Ryu JK, Han SA, Won KY. Solitary fibrous tumor of the breast: a case report and review of the literature. J Med Ultrason (2001). 2016;43(1):125-8. doi: 10.1007/s10396-015-0674-9. [PubMed: 26703178].

5. Rovera F, Imbriglio G, Limonta G, Marelli M, La Rosa S, Sessa F, et al. Solitary fibrous tumor of the male breast: a case report and review of the literature. World J Surg Oncol. 2008;6:16. doi: 10.1186/1477-7819-6-16. [PubMed: 18254983]. [PubMed Central: PMC2266749].
6. Tsai SY, Hsu CY, Chou YH, Lai YC, Lin YH, Wang HK, et al. Solitary fibrous tumor of the breast: A case report and review of the literature. J Clin Ultrasound. 2017;45(6):350-4. doi: 10.1002/jcu.22415. [PubMed: 27753108].

7. Yang LH, Dai SD, Li QC, Xu HT, Jiang GY, Zhang Y, et al. Malignant solitary fibrous tumor of breast: a rare case report. Int J Clin Exp Pathol. 2014;7(7):4461-6. [PubMed: 25120834]. [PubMed Central: PMC4129069].

8. Magro G, Angelico G, Leone G, Palazzo J. Solitary fibrous tumor of the breast: report of a case with emphasis on diagnostic role of STAT6 immunostaining. Pathol Res Pract. 2016;212(5):463-7. doi: 10.1016/j.prp.2015.12.013. [PubMed: 26778386].

9. Meguerditchian AN, Malik DA, Hicks DG, Kulkarni S. Solitary fibrous tumor of the breast and mammary myofibroblastoma: the same lesion? Breast J. 2008;14(3):287-92. doi: 10.1111/j.1524-4741.2008.00588.x. [PubMed: 18384484].

10. Ge W, Yu DC, Chen G, Ding YT. Clinical analysis of 47 cases of solitary fibrous tumor. Oncol Lett. 2016;12(4):2475-80. doi:10.3892/ol.2016.4967. [PubMed: 27698815]. [PubMed Central: PMC5038456].

11. Barr RG, Nakashima K, Amy D, Cosgrove D, Farrokh A, Schafer F, et al. WFUMB guidelines and recommendations for clinical use of ultrasound elastography: Part 2: breast. Ultrasound Med Biol. 2015;41(5):1148-60. doi: 10.1016/j.ultrasmedbio.2015.03.008. [PubMed: 25795620]

12. Doyle LA, Vivero M, Fletcher CD, Mertens F, Hornick JL. Nuclear expression of STAT6 distinguishes solitary fibrous tumor from histologic mimics. Mod Pathol. 2014;27(3):390-5. doi: 10.1038/modpathol.2013.164. [PubMed: 24030747].

13. Riola-Parada C, Jimenez-Ballve A, Serrano-Palacio A, Cabrera-Martin MN, Brenes-Sanchez JM, Carreras-Delgado JL. Solitary fibrous breast tumour: Contribution of (18)F-FDG PET/CT. Rev Esp Med Nucl Imagen Mol. 2017;36(1):61-2. doi: 10.1016/j.remn.2016.01.002. [PubMed: 26897461]. 